\title{
Cortico-Thalamic Circuit Model for Bottom-Up and Top-Down Mechanisms in General Anesthesia Involving the Reticular Activating System
}

\author{
Axel Hutt ${ }^{1, *}$ \\ ${ }^{1}$ Department of Data Assimilation, German Weather Service, Offenbach, Germany \\ "Corresponding author: Department of Data Assimilation, German Weather Service, Offenbach, Germany. Email: digitalesbad@gmail.com \\ Received 2019 June 18; Accepted 2019 June 26.
}

\begin{abstract}
General anesthesia is a widely used medical procedure. However, its underlying physiological mechanisms are still unknown. Current research has identified bottom-up mechanisms in the brain involving subcortical sleep-promoting and arousal structures and top-down mechanisms comprising corticocortical and corticothalamic circuits. The current work presents a neural model considering both mechanisms. Its numerical simulation yields frontal and occipital cortical activity that exhibits the characteristic spectral changes as observed experimentally. In addition, increasing the anesthetic level enhances local synchrony and weakens distant synchrony. This represents a fragmentation of the brain as observed in experimental data under anesthesia.
\end{abstract}

Keywords: Anesthesia, Loss of Consciousness, Electroencephalogram, Neural Model

\section{Context}

General anesthesia is an omnipresent medical procedure in hospital practice. Today, general anesthesia is recognized as a safe medical procedure although it may exhibit severe side effects such as postoperative confusion or nausea and vomiting. One reason for the missing control over these effects is the limited understanding of the systemic physiological mechanisms of anesthetic actions and how these are linked to physiological outcomes. A prominent effect of most of the clinically relevant anesthetic drugs is the characteristic spectral change in the electrophysiological activity across cortical brain areas over multiple frequency bands $(1,2)$. A prominent spectral change observed is the frequent induction of oscillatory activity in the $\beta$-frequency band complemented by a subsequent decrease to the $\alpha$-frequency band with increasing anesthetic concentration. This spectral change correlates strongly with the patients' behavioral state, e.g., changes from a sedated state to loss of consciousness (LOC).

To better understand the relationship between the behavioral state change on a macroscopic description scale and physiological action on a microscopic scale, it is reasonable to describe the brain dynamics by a mesoscopic abstract model that links the two description levels. For in- stance, a mesoscopic system model describes effective neural action as bottom-up and top-down anesthetic mechanisms $(3,4)$. The bottom-up mechanism considers anesthetic action on subcortical areas, such as the hypothalamus and the brainstem. The anesthetic action in sleeppromoting and arousal-promoting regions is recognized to play an important role in the depression of brain functions and LOC. The top-down mechanism recognizes corticocortical and thalamocortical connectivity as the primary feature in the understanding of LOC. For instance, anesthetic agents are known to affect synaptic receptors in cortical neurons to disturb the interaction with thalamic structures that, in turn, induces sedation and LOC. For more details, one can refer to the excellent article by Mashour and Hudetz (3). To implement numerically such mechanisms, we propose to utilize a neural population model (5-7) that captures essential microscopic action and describes macroscopic dynamics.

In our model (8), we consider a circuit between the cortex and thalamic structures. This circuit is driven by fluctuating input from the reticular activating system (RAS) that sets the level of excitation in the brain $(3,8)$. This input represents the bottom-up mechanism while anesthetic action on the RAS is modeled as a variable intensity of ran- 
dom fluctuation. In addition, anesthetic agents are known to affect synaptic receptors in the thalamus and the cortex. We model this synaptic action in a mesoscopic approach. This model of synaptic cortical and thalamic action represents the top-down mechanism. In a previous work (8), we showed in detail that the combination of both mechanisms is essential to describe a large number of wellknown observations.

The subsequent section introduces briefly the computational model, followed by simulation results. A final discussion puts the results into context and embeds them into the current literature.

\section{Evidence Acquisition}

\subsection{Bottom-Up Mechanism: The Reticular Activating System}

Anesthetic agents are known to affect neural information processing by modification of ion channel gates or synaptic receptor properties $(9,10)$. Specifically, anesthetics reduce both cortical input from the thalamus and thalamic and cortical firing activity. This originates from inhibition in the RAS $(3,8)$. By virtue of the complexity of the input to the cortex and thalamus and since it is not known in detail, an initial approach considers this external input to the brain as fluctuations, which are random in time and space. The proposed model takes into account neural population activity and random fluctuations model uncorrelated synaptic input to target neurons in the cortex and thalamus with a high population firing rate.

Clinically relevant anesthetics affect GABAergic extrasynaptic and synaptic receptors in the RAS $(5,11)$. GABAergic synaptic receptors are present in the hypothalamus, basal forebrain, and the brainstem $(12,13)$, which are part of the RAS. There the anesthetics prolonged phasic responses at synaptic receptors. Extra-synaptic receptors are present in the brainstem, hypothalamus, and thalamus $(5,11,14)$ and anesthetics enhance their tonic inhibitory current. It is important to mention that extra-synaptic receptors are rather sensitive to anesthetic agents (15) and hence, they probably play an important role in the understanding of LOC.

\subsection{Top-Down Mechanism: Anesthetic Effect Outside the RAS}

In addition to the effect of fluctuations, a large number of anesthetics induce inhibition by modifying cortical and thalamic GABAergic synaptic receptors. To model this synaptic effect, the model considers a synaptic decay time scale prolongation of inhibitory GABAergic receptors
$(7,16)$ while retaining constant the maximum synaptic impulse response.

\subsection{Bottom-Up and Top-Down Mechanism in a Single Dynamic Model}

For a deeper understanding of the underlying neural mechanism of electrophysiological activity changes, we developed a thalamocortical network model including both the frontal and the occipital cortex (Figure 1).

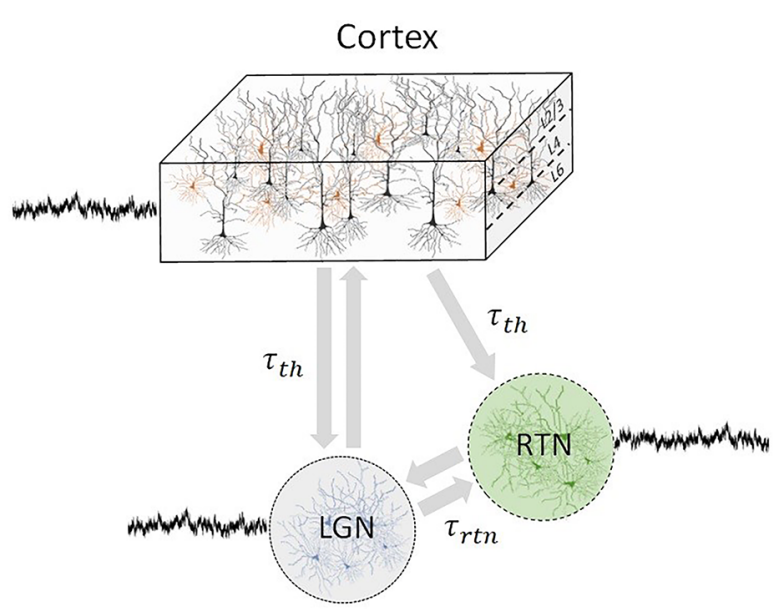

Figure 1. Illustration of thalamocortical network topology subjected to additive fluctuations involving the cortex, lateral geniculate nucleus (LGN), and reticular thalamic nucleus (RTN). Connections are delayed with $\tau_{\text {th }}$ and $\tau_{\text {rtn }}$. Here, the cortex may be the frontal or the occipital cortex, both of which are independent of each other. For either case model parameters are different (8).

Previous theoretical studies have shown that it is sufficient to consider the relationship between excitation and inhibition in the system to explain major spectral changes in the experimental electroencephalogram (EEG) under anesthesia $(7,17)$. The network comprises delayed recurrently connected neural ensembles of inhibitory interneurons, excitatory pyramidal neurons, thalamic reticular neurons (RTN) and thalamic lateral geniculate nucleus (LGN) relay neurons. More details on the model are presented elsewhere (8).

\section{Results}

Numerical simulations of the neural model provide cortical excitatory and inhibitory mean potentials, as well as mean potentials in the LGN and RTN. Moreover, the model comprises spiking activity in each neural population that allows, inter alia, to study the spike field coherence between populations. The cortex may be the frontal 
cortex or the occipital cortex, for which the model parameters are different.

\subsection{Bottom-Up Fluctuations}

The RAS comprises a complex network of inhibitory neural structures. Anesthetic agents may increase the synaptic time constant, decrease the pre-synaptic firing rate, and causes a decrease in the synapse's phasic response. In addition, anesthetic agents are known to increase the tonic inhibitory current in GABAergic extrasynaptic receptors $(5,11,14)$.

Figure 2A demonstrates in model simulations (8) that a strong anesthetic action decreases the mean level and magnitude of RAS activity, i.e. the input to the corticothalamic circuit. Figure 2B summarizes these results showing the mean and standard deviation of fluctuations subjected to the anesthetic level. This result agrees with experimental evidence in anesthetized rats and humans (18). The corresponding study shows that, in the isoelectric state, primary and somatosensory neurons are fully responsive to external stimuli. Such isoelectric states occur under deep anesthesia. Hence, this study indicates that neurons are not strongly inhibited in deep anesthesia, which contrasts to the hypothesis that deep anesthesia merely yields synaptic inhibition. Consequently, anesthetic action diminishes the input from RAS to the neurons while retaining the neurons' responsiveness.

The proposed model does not specify in detail the relationship between anesthetic action and RAS fluctuation intensity. We hypothesize that this relationship is an effective mesoscopic description of physiological actions of anesthetics. This effective description may be powerful in the light of complex interactions between different drugs and hence, it is valid for the combinations of drugs. We hypothesize that this effective action represents one of the main bottom-up mechanisms of anesthetics in thalamocortical populations.

\subsection{Simulated EEG}

In order to understand the bottom-up and top-down mechanisms role of RAS and synaptic anesthetic action in anesthesia, we simulated the thalamocortical neural model described above. Modifying the RAS fluctuation variance and the synaptic inhibition effect over time, we gained the temporal evolution of the simulated EEG spectral density seen in Figure 3. We found that the model exhibits an emerging $\beta$-band activity with a subsequent rhythmic slowing-down fluctuation intensity and increasing anesthetic synaptic effect, as would occur when the concentration of anesthetic drugs increases. This deceleration represents a transition from the $\beta$-band activity to $\alpha$ activity. A subsequent increase of the fluctuation level and decrease of the anesthetic synaptic effect invert the characteristic spectral features. This shape of the power spectrum reproduces well experimental data observed in frontal EEG electrodes with the characteristic anesthetic smile $(8,19)$.

Figure 4 shows the simulation results of occipital EEG. We observe an initial strong $\alpha$ oscillation well-known for closed eyes (cf. Figure 4A). For decreasing fluctuation level and excitatory input to the cortical excitatory population and increasing anesthetic synaptic effect, the $\alpha$ oscillation drops sharply in intensity and almost vanishes. This is in close agreement with experimental findings (16). Here, the change in the fluctuation intensity and the excitatory input is part of the bottom-up mechanism while the synaptic effect reflects the top-down mechanism effect.

\subsection{Intra-Area and Inter-Area Synchronization}

To better illustrate intra-cortical dynamics subjected to the bottom-up and top-down mechanisms, Figures 5A and $5 \mathrm{~B}$ show the simulated intra-area phase synchronization of dendritic activity between frontal and occipital cortical excitatory cells, respectively. For the frontal area seen in Figure $5 \mathrm{~A}$, we observe a monotonic phase synchronization enhancement with decreasing fluctuation level and increasing the anesthetic synaptic effect. This is in good agreement with the current knowledge that frontal EEG (and hence frontal cortical population activity) is rather asynchronous in the awake state (without anesthetic action) and synchronous in the sedation phase and under deeper anesthesia (20). In contrast, the phase synchronization of the occipital cortex (Figure 5B) remains high for the whole period. This contrasts to experimental EEG findings (20) showing a drop of phase coherence in occipital electrodes. This indicates a weak point in the model, which will be addressed in future work.

Phase synchrony reflects functional connectivity between neurons and their ensembles. In addition to intraarea synchronization seen in Figures $5 \mathrm{~A}$ and 5B, Figures $5 \mathrm{C}$ and $5 \mathrm{D}$ show the cross spike-field coherence ( 8 ) between the cortex and thalamic areas. Increasing the anesthetic action in the bottom-up and top-down mechanisms eliminates the coherence between cortical and thalamic cells in the full frequency spectrum. Hence, the thalamus and cortex decouple functionally for weaker bottom-up fluctuations and enhanced synaptic anesthetic action. 

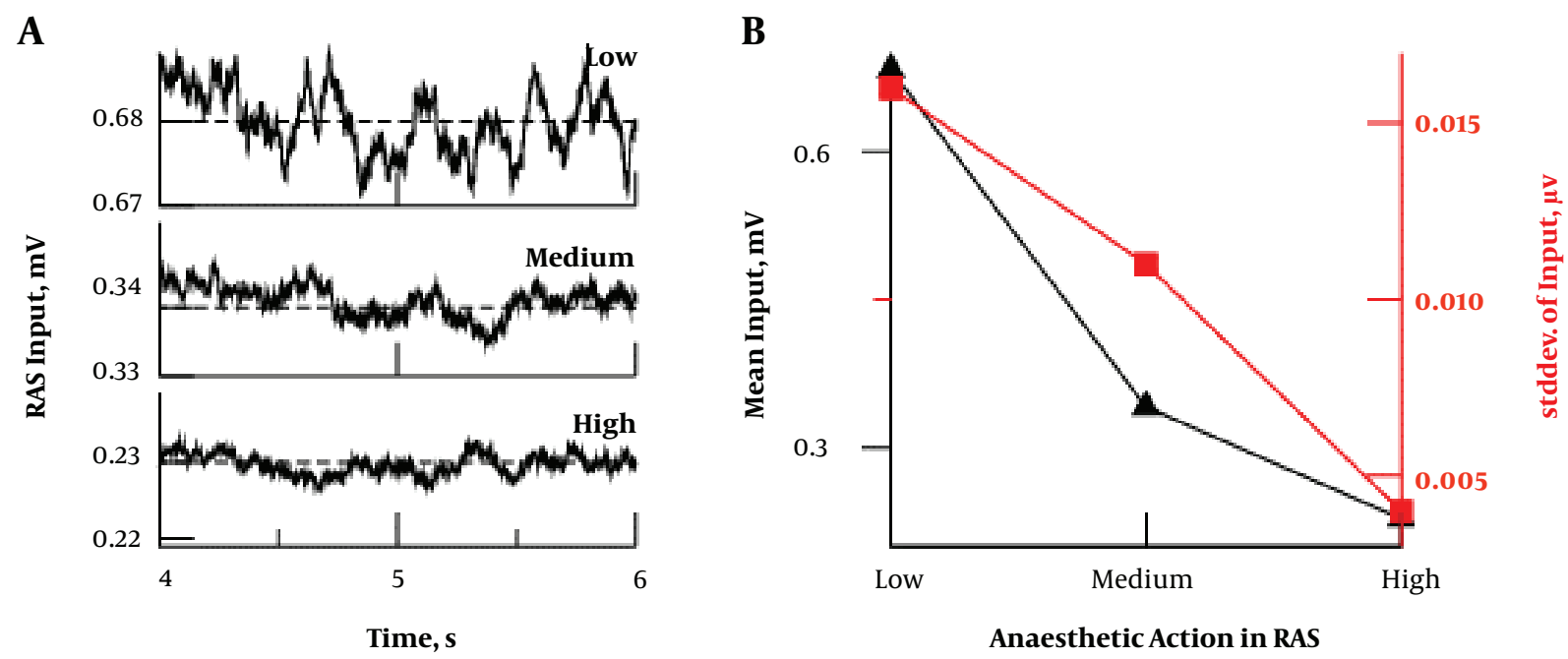

Figure 2. Synaptic and extra-synaptic effects on the RAS input to cortical and thalamic activity (8); A, increasing anesthetic levels reduce the amplitude of excitatory mean potentials in the RAS; B, mean and standard deviation of RAS input decreases with increasing anesthetic level.

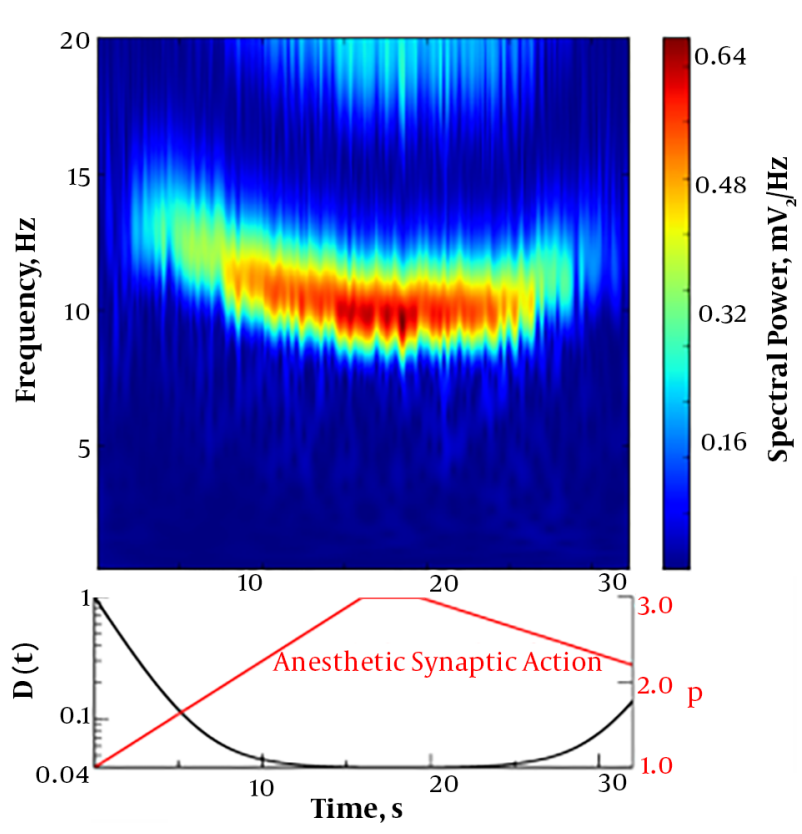

Figure 3. Numerical simulation of frontal EEG; spectral power density (top panel), fluctuation level $\mathrm{D}$ and anesthetic synaptic effect $\mathrm{p}$ (lower panel)

\section{Conclusions}

The model presented here describes the well-known characteristic spectral changes in EEG (19-21), the enhancement of local intra-area synchrony (20), and loss of interarea synchrony $(22,23)$. Our work introduces the bottomup notion of a general decrease in neural fluctuations orig- inating in the RAS that tunes the anesthetic-induced behavioral transitions. Hence, weaker RAS input reduces the neural information flow between neural structures and separates functionally connected networks. In summary, our results support the importance of fluctuating input from the RAS as the bottom-up mechanism. Nevertheless, only the combination of RAS input and synaptic anesthetic action provide a coherent description of brain dynamics (8).

The current work investigated the dynamics in the frontal and occipital cortex, but neglected other brain areas that are affected by anesthetics, such as the default network (24) or the parietal cortex (25). Moreover, our work showed the effects in light anesthesia but neglected electrophysiological features found under deep anesthesia, such as intra-area fragmentation (24), slow frequencies $<1 \mathrm{~Hz}$ (19) or burst suppression (26).

\section{Footnotes}

Conflicts of Interests: There are no financial conflicts of interest to disclose.

Funding/Support: No funding has been provided. 
A

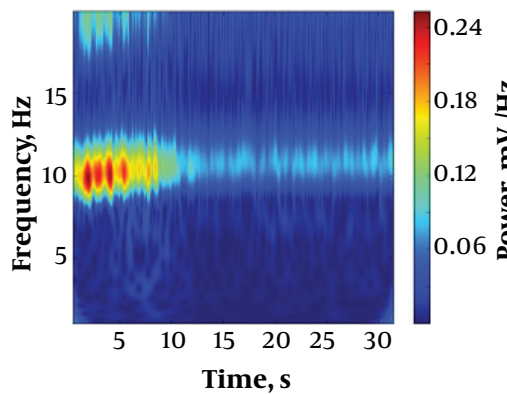

B

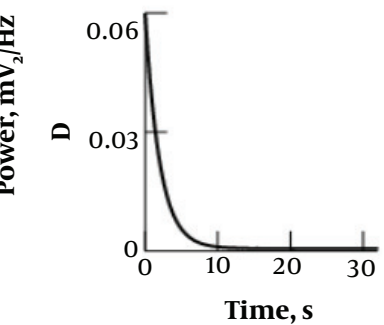

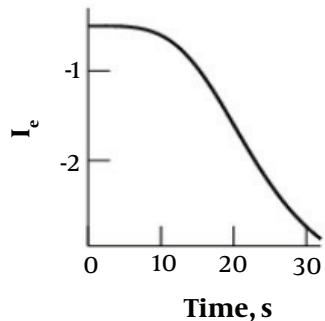

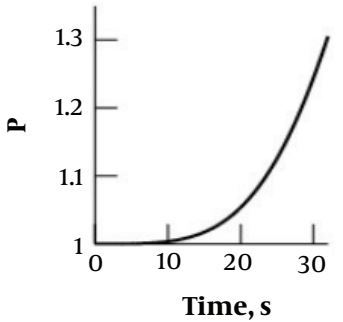

Figure 4. Numerical simulation of occipital EEG; A, spectral power density; B, fluctuation intensity D, excitatory input to the cortical excitatory population $I_{\mathrm{e}}$ and the anesthetic synaptic effect as a factor of the synaptic decay time $\mathrm{p}$, see (8) for more details.

A

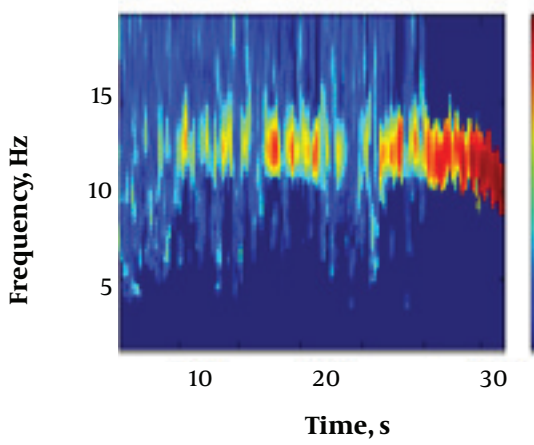

C

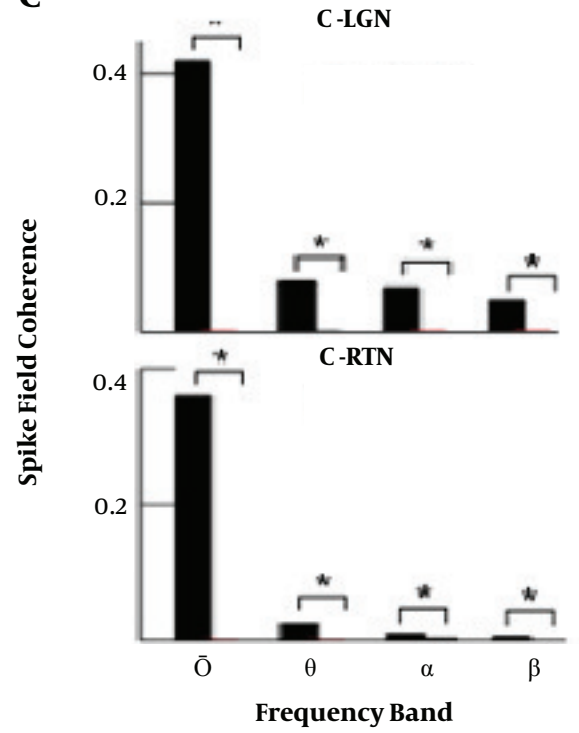

B
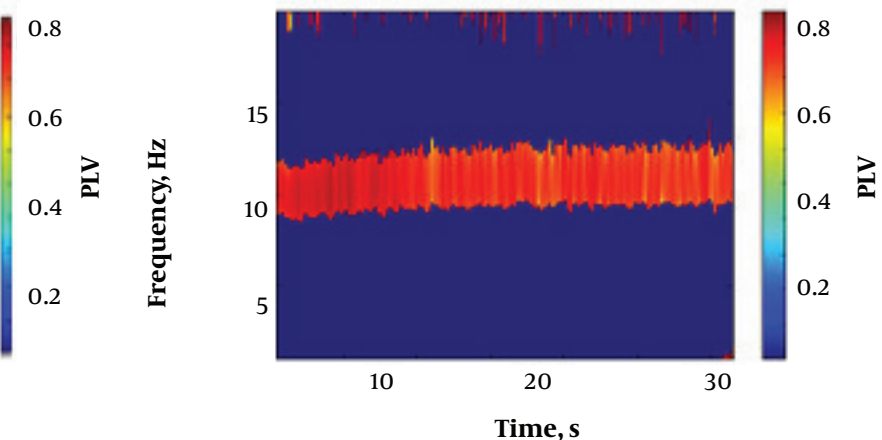

D

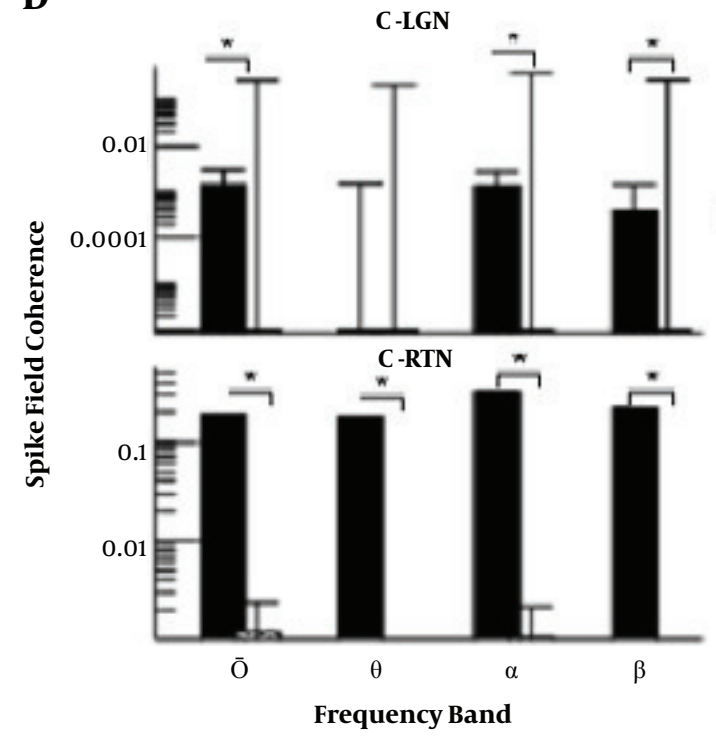

Figure 5. Intra-area and inter-area synchronization in the frontal and occipital cortex; A and B are phase locking value (PLV) in intra-cortical frontal and occipital neural population, respectively. The RAS input and anesthetic synaptic action are taken from Figures 3 and 4 ; C, inter-area SFC between frontal cortical and thalamic populations; D, inter-area SFC between occipital cortical and thalamic populations. For both panels in C and D, the solid bar denotes SFC for D $(t=0 \mathrm{~s})$ and the dashed bar SFC for $\mathrm{D}(\mathrm{t}=30 \mathrm{~s}$ ). 


\section{References}

1. Sellers KK, Bennett DV, Hutt A, Williams JH, Frohlich F. Awake vs. anesthetized: Layer-specific sensory processing in visual cortex and functional connectivity between cortical areas. J Neurophysiol. 2015;113(10):3798-815. doi: 10.1152/jn.00923.2014. [PubMed: 25833839]. [PubMed Central: PMC4473519].

2. Barttfeld P, Uhrig L, Sitt JD, Sigman M, Jarraya B, Dehaene S. Signature of consciousness in the dynamics of resting-state brain activity. Proc Natl Acad Sci U S A. 2015;112(3):887-92. doi:10.1073/pnas.1418031112. [PubMed: 25561541]. [PubMed Central: PMC4311826].

3. Mashour GA, Hudetz AG. Bottom-up and top-down mechanisms of general anesthetics modulate different dimensions of consciousness. Front Neural Circuits. 2017;11:44. doi: 10.3389/fncir.2017.00044. [PubMed: 28676745]. [PubMed Central: PMC5476707].

4. Mashour GA, Hudetz AG. Fading whispers down the lane: Signal propagation in anaesthetized cortical networks. Br J Anaesth. 2017;119(4):568-70. doi: 10.1093/bja/aex215. [PubMed: 29121276].

5. Hutt A, Buhry L. Study of GABAergic extra-synaptic tonic inhibition in single neurons and neural populations by traversing neural scales: Application to propofol-induced anaesthesia. J Comput Neurosci. 2014;37(3):417-37. doi: 10.1007/s10827-014-0512-x. [PubMed: 24976146]. [PubMed Central: PMC4224752].

6. Hutt A, Hashemi M, beim Graben P. How to render neural fields more realistic. In: Bhattacharya B, Chowdury FN, editors. Validating neural computational models of neurological and psychiatric disorders. Springer; 2015. p. 141-59. doi: 10.1007/978-3-319-20037-8_6.

7. Steyn-Ross DA, Steyn-Ross ML, Sleigh JW, Wilson MT. Progress in modeling EEG effects of general anesthesia: Biphasic response and hysteresis. In: Hutt A, editor. Sleep and anesthesia: Neural correlates in theory and experiments. Springer; 2011. p. 167-94. doi: 10.1007/978-1-46140173-5_8.

8. Hutt A, Lefebvre J, Hight D, Sleigh J. Suppression of underlying neuronal fluctuations mediates EEG slowing during general anaesthesia. Neuroimage. 2018;179:414-28. doi: 10.1016/j.neuroimage.2018.06.043. [PubMed: 29920378].

9. Alkire MT, Miller J. General anesthesia and the neural correlates of consciousness. Prog Brain Res. 2005;150:229-44. doi: 10.1016/S00796123(05)50017-7. [PubMed: 16186027].

10. Brown EN, Lydic R, Schiff ND. General anesthesia, sleep, and coma. $N$ Engl J Med. 2010;363(27):2638-50. doi: 10.1056/NEJMra0808281. [PubMed: 21190458]. [PubMed Central: PMC3162622].

11. Kopanitsa MV. Extrasynaptic receptors of neurotransmitters: Distribution, mechanisms of activation, and physiological role. Neurophysiology.1997;29(6):357-65. doi:10.1007/bf02463356.

12. Alkire MT, Hudetz AG, Tononi G. Consciousness and anesthesia. Science. 2008;322(5903):876-80. doi: 10.1126/science.1149213. [PubMed: 18988836]. [PubMed Central: PMC2743249].

13. Franks NP. General anaesthesia: From molecular targets to neuronal pathways of sleep and arousal.Nat Rev Neurosci.2008;9(5):370-86. doi: 10.1038/nrn2372. [PubMed: 18425091].

14. Hashemi M, Hutt A, Sleigh J. Anesthetic action on extra-synaptic receptors: Effects in neural population models of EEG activity. Front Syst Neurosci. 2014;8:232. doi: 10.3389/fnsys.2014.00232. [PubMed: 25540612]. [PubMed Central: PMC4261904].
15. McDougall SJ, Bailey TW, Mendelowitz D, Andresen MC. Propofol enhances both tonic and phasic inhibitory currents in second-order neurons of the solitary tract nucleus (NTS). Neuropharmacology. 2008;54(3):552-63. doi: 10.1016/j.neuropharm.2007.11.001. [PubMed: 18082229]. [PubMed Central: PMC2351956].

16. Hashemi M, Hutt A, Sleigh J. How the cortico-thalamic feedback affects the EEG power spectrum over frontal and occipital regions during propofol-induced sedation. J Comput Neurosci. 2015;39(2):155-79. doi: 10.1007/s10827-015-0569-1. [PubMed: 26256583].

17. Hutt A. The anesthetic propofol shifts the frequency of maximum spectral power in EEG during general anesthesia: analytical insights from a linear model. Front Comput Neurosci. 2013;7:2. doi 10.3389/fncom.2013.00002. [PubMed: 23386826]. [PubMed Central: PMC3564209].

18. Altwegg-Boussac T, Schramm AE, Ballestero J, Grosselin F, Chavez M, Lecas S, et al. Cortical neurons and networks are dormant but fully responsive during isoelectric brain state. Brain. 2017;140(9):2381-98. doi: 10.1093/brain/awx175. [PubMed: 29050394].

19. Purdon PL, Pierce ET, Mukamel EA, Prerau MJ, Walsh JL, Wong KF et al. Electroencephalogram signatures of loss and recovery of consciousness from propofol. Proc Natl Acad Sci U S A. 2013;110(12):E114251. doi:10.1073/pnas.1221180110. [PubMed: 23487781]. [PubMed Central: PMC3607036].

20. Cimenser A, Purdon PL, Pierce ET, Walsh JL, Salazar-Gomez AF, Harrell PG, et al. Tracking brain states under general anesthesia by using global coherence analysis. Proc Natl Acad Sci U S A. 2011;108(21):88327. doi: 10.1073/pnas.1017041108. [PubMed: 21555565]. [PubMed Central: PMC3102391].

21. Sellers KK, Bennett DV, Hutt A, Frohlich F. Anesthesia differentially modulates spontaneous network dynamics by cortical area and layer. J Neurophysiol. 2013;110(12):2739-51. doi: 10.1152/jn.00404.2013. [PubMed: 24047911].

22. Massimini M, Ferrarelli F, Huber R, Esser SK, Singh H, Tononi G. Breakdown of cortical effective connectivity during sleep. Science. 2005;309(5744):2228-32. doi: 10.1126/science.1117256. [PubMed: 16195466].

23. Wollstadt P, Sellers KK, Rudelt L, Priesemann V, Hutt A, Frohlich F, et al. Breakdown of local information processing may underlie isoflurane anesthesia effects. PLoS Comput Biol. 2017;13(6). e1005511. doi: 10.1371/journal.pcbi.1005511. [PubMed: 28570661]. [PubMed Central: PMC5453425].

24. Huang Z, Liu X, Mashour GA, Hudetz AG. Timescales of intrinsic bold signal dynamics and functional connectivity in pharmacologic and neuropathologic states of unconsciousness. J Neurosci. 2018;38(9):2304-17. doi: 10.1523/JNEUROSCI.2545-17.2018. [PubMed: 29386261]. [PubMed Central: PMC5830518].

25. Pal D, Dean JG, Liu T, Li D, Watson CJ, Hudetz AG, et al. Differential role of prefrontal and parietal cortices in controlling level of consciousness. Curr Biol. 2018;28(13):2145-52 e5. doi: 10.1016/j.cub.2018.05.025. [PubMed: 29937348]. [PubMed Central: PMC6039257].

26. Ching S, Purdon PL, Vijayan S, Kopell NJ, Brown EN. A neurophysiological-metabolic model for burst suppression. Proc Natl Acad Sci U S A. 2012;109(8):3095-100. doi: 10.1073/pnas.1121461109. [PubMed: 22323592]. [PubMed Central: PMC3286963]. 